\title{
Identification and Control Using Direction Basis Function Neural Network
}

\author{
Mahdi Jalili-Kharaajoo \\ Young Researchers Club, Azad University, Tehran, Iran \\ mahdijalili@ece.ut.ac.ir
}

\begin{abstract}
In this paper, adaptive identification and control of nonlinear dynamical systems are investigated using Two Synaptic Weight Neural Networks (TSWNN). The identification algorithm has the properties of rapid convergence and persistent adaptability that make it suitable for real-time control. A nonlinear example is simulated to demonstrate the effectiveness of the identification and control algorithms.
\end{abstract}

\section{Introduction}

Similar to the Multilayer Feedforward Neural Networks (MFNN), Two Synaptic Weight Neural Networks (TSWNN) possesses the capacity of universally approximating nonlinear multi-variable functions. An n-means clustering technique was regarded as a better method for updating the kernels by Feng Cao [1]. For on-line and adaptive applications of neural network model, some kinds of recursive identification algorithms are naturally required [2-5].

In this paper, the kernels and weights of the TSWNN are also updated simultaneously. A novel approach, however, is presented to train the TSWNN. Specifically, an Adaptive Fuzzy Generalized Learning Vector Quantization (AFGLVQ) technique is adopted to update the kernels, its weights and recursive least squares algorithm with variable forgetting factor (VRLS) is used to estimate the weights.

\section{Two Synaptic Weight Neural Networks for Modeling Nonlinear System}

Many single-input single-output non-linear systems can be described as

$$
y(t)=f_{s}\left(y(t-1), \ldots, y\left(t-n_{y}\right), u(t-1), \ldots, u\left(t-n_{u}\right)\right)
$$

where $y(t)$ and $u(t)$ are the system output and input, respectively; $n_{y}$ and $n_{u}$ the lags of the output and input respectively; and $f_{s}($.) some non-linear function. The TSWNN is a two-layer processing structure. In the first layer, the neurons are represented by TSWNN with the kernels $c_{i}$, which are interconnected by weights $w_{i j}, w_{j i}^{\prime}$. Factor $s, p$ is chosen. The second layer is essentially a linear combiner.

The overall response of such a network is a mapping $f_{r}: R^{m} \rightarrow R$, that is 


$$
f_{r}(x)=\sum_{i=1}^{n} \theta_{i} \phi\left(x, c_{i}, w_{i}\right)
$$

where $x \in R^{m}, m$ is network input vector, $c_{i} \in R^{M}, 1 \leq i \leq n$ are the kernels and $\phi():. R \rightarrow R . \theta_{i}$ is the connection weights; and $n$ is the number of neurons. When the TSWNN is used to approximate the dynamical system (1), define $m=n y+n u$ and let

$$
x(t)=\left[y(t-1), \ldots, y\left(t-n_{y}\right) ; u(t-1), \ldots, u\left(t-n_{u}\right)\right]
$$

Then the TSWNN output

$$
\hat{y}(t)=f_{r}(x(t))
$$

acts as the estimator of the $y(t)$. Let

$$
e(t)=\hat{y}(t)-y(t)
$$

Hence, the goal of training the TSWNN is to make $e(t)$ as small as possible. In this paper, the function is chosen as function

$$
\phi\left(x, c_{i}, w_{i}\right)=\cos \left[\sum_{j=1}^{m}\left(\frac{w_{j}}{\left|w_{j}\right|}\right)^{s}\left|w_{j}\left(X_{j}-c_{j}\right)\right|^{p}-\theta\right]
$$

\section{Hybrid AFGLVQ and VRLS Algorithm}

\subsection{Adaptive Fuzzy Generalized Learning Vector Quantization (FGLVQ)}

\subsubsection{FGLVQ}

In the GLVQ algorithm, for the winner node $i\left(i=\arg \min \left|x(t)-c_{j}(t)\right|, 1<i<n\right)$, the updating rules of the kernels and its weights are

$$
c_{i}(t+1)=c_{i}(t)+b(t)\left[\frac{D^{2}-D+\left\|x(t)-c_{i}(t)\right\|}{D^{2}}\right]\left(x(t)-c_{i}(t)\right), w_{i}(t+1)=w_{i}(t)+\eta_{o}\left\|x(t)-c_{i}(t)\right\|\left(x(t)-c_{i}(t)\right)
$$

For the rest nodes $j(j=1, \ldots, n, j \neq i)$, the rules are

$$
c_{j}(t+1)=c_{j}(t)+b(t)\left[\frac{D^{2}-D+\left\|x(t)-c_{j}(t)\right\|}{D^{2}}\right]\left(x(t)-c_{j}(t)\right), w_{j}(t+1)=w_{j}(t)+\eta_{o}\left\|x(t)-c_{j}(t)\right\|\left(x(t)-c_{j}(t)\right)
$$

where $D=\sum_{i=1}^{n}\left\|x(t)-c_{i}(t)\right\|, \eta_{o}, \eta_{1} \quad 1$ is proportion factor; $b(t)$ is the learning rate which is chosen satisfy the two conditions: As $t \rightarrow \infty ; b(t) \rightarrow 0$ and $b(t) \rightarrow \infty . b(t)$ can be taken as

$$
b(t)=b(t-1) / \sqrt{1+t / n}
$$

The algorithm can give more reasonable solution when $D>1$. But it has been found that GLVQ behaves exactly opposite to what was desired when $D<1$. To overcome this problem, we can give a fuzzy modification of GLVQ. Let $L_{x}$ be a loss function which measures the locally weighted mismatch (error) with respect to the winner

$$
L_{x}=L\left(x, c_{1}, c_{2}, \ldots, c_{n}\right)=\sum_{j=1}^{n} \mu_{j}\left\|x-c_{j}\right\|^{2}
$$


where $\mu_{j}$ is chosen to be a fuzzy member function as

$$
\mu_{j}=\left(\sum_{p=1}^{n} \frac{\left\|x-c_{j}\right\|^{2}}{\left\|x-c_{p}\right\|^{2}}\right)^{-1}
$$

Assume $\left\|x-c_{p}\right\|>0$, the gradient of $L_{x}$ with respect to $c_{p}$ can be calculated as

$$
\nabla_{c_{p}} L=-2 n \mu_{p}^{2}\left(x-c_{p}\right)
$$

Thus, the updating equation of the kernels can be rewritten as

$$
c_{j}(t+1)=c_{j}(t)+2 b(t) n \mu_{j}^{2}\left(x(t)-c_{j}(t)\right)
$$

\subsubsection{Adaptive Modification of FGLVQ}

In adaptive identification and control, since a plant is unknown and the structure of a given TSWNN is limited, therefore the modeling error exists inevitably. Here we give an updating equation of $b(t)$

$$
b(t)=b_{o}\|\mathcal{E}(t)\| /(1+\|\varepsilon(t)\|)
$$

where $0<b_{o}<1$, thus $0<b(t)<1 . b(t)$ varies with $\varepsilon(t)$. Of course, when $\varepsilon(t)$ converges to zero, $b(t)$ will tend to zero too.

\subsection{Recursive Least Squares with Variable Forgetting Factor}

Define the hidden layer output vector at the instant $t$ as

$$
\Phi_{t}=\left[\phi_{1}(t), \phi_{2}(t), \ldots, \phi_{n}(t)\right]^{T}
$$

The connection weight vector at $t$ as

$$
\Theta_{t}=\left[\theta_{1}(t), \theta_{2}(t), \ldots, \theta_{n}(t)\right]^{T}
$$

Thus, $\Theta_{t}$ can be recursively updated by the equations

$$
\Theta_{t+1}=\Theta_{t}+K_{t} \varepsilon(t), K_{t}=\frac{P_{t} \Phi_{t}}{1+\Phi_{t}^{T} P \Phi_{t}}, P_{t+1}=\left(I-K_{t} \Phi_{t}^{T}\right) P_{t} / \rho_{t}, \rho_{t}=1-\left(1+\Phi_{t}^{T} K_{t}\right) \varepsilon_{t}^{2} / \sum_{o}
$$

If $\rho_{t}<\rho_{\min }$, set $\rho_{t}=\rho_{\min } ; \sum_{o}>0$ reflects the amplitude of the noise.

\section{Adaptive Control Law Optimization}

Define the predictive control error

$$
e(t+1)=\hat{y}(t+1)-\left[\alpha y(t)+(1-\alpha) y_{r}(t+1)\right]
$$

where $y_{r}(t+1)$ is the reference output. Let the initial value of $u(t)$ be

$$
\mu_{o}(t)=\mu(t-1)
$$

and the corresponding output of the TSWNN be

$$
\hat{y}_{o}(t+1)=f_{r}\left(x_{o}(t+1)\right) ; \quad x_{o}(t+1)=\left[y(t), \ldots, y\left(t-n_{y}+1\right) ; u(t), \ldots, u\left(t-n_{u}+1\right)\right]
$$

Similarly, $u_{k}(t)$ denotes the control value. The corresponding output of the TSWNN is

$$
\left.\hat{y}_{k}(t+1)=f_{r}\left(x_{k}(t+1)\right) ; x_{k}(t+1)=[y(t), \ldots y)\left(t-n_{y}+1\right) ; u_{k}(t), \ldots u_{k}\left(t-n_{u}+1\right)\right], e_{k}(t+1)=\hat{y}(t+1)-\left[\alpha y(t)+(1-\alpha) y_{r}(t+1)\right]
$$


The optimization procedure is described as follows.

- Let $k_{\max }$ be the maximum iteration number and $e_{\max }$ be the desired control accuracy. Set the initial Hessian matrix $H_{o}=h_{o} I, 0<h_{o}<1, k=0$.

- Let $k=k+1 . u(t)$ at the $k$ iteration is calculated by

$$
\mu_{k}(t)=\mu_{k-1}(t)-H_{k} \nabla \hat{y}_{k-1} e_{k-1}, H_{k}=\left(\lambda_{c} H_{k-1}^{-1}+\nabla^{T} \hat{y}_{k-1}+\alpha_{c} I\right)^{-1}
$$

where $0<\lambda_{c}<1,0<\alpha_{c}<1 ; \nabla \hat{y}_{k-1}$ is the gradient of $y_{k-1}(t+1)$ with respect to $u_{k-1}(t)$.

- If $k=k_{\max }$ or $e_{k}(t+1)=e_{\max }$, then stop calculating; Otherwise, go to the previous step. In the DLS algorithm, the updating equation for $H_{k}$ is

$$
H_{k}=\left(\lambda_{c} H_{k-1}^{-1}+\nabla \hat{y}_{k-1} \nabla^{T} \hat{y}_{k-1}\right)^{-1}
$$

The item $\alpha_{c} I$ of the equation (22) is not included in the equation (23). It has been found that when $\hat{y}_{k-1}$ is small and $0<\lambda_{c}<10$, the $H_{k}$ will become divergent infinitely as $k$ increases.

\section{Simulation Results}

Consider $\quad y(k)=\frac{y(k-10)}{1+y^{2}(k-1)}+u^{3}(k-1) ; \quad m=7, h_{o}=0.09, b_{o}=0.21, \lambda_{c}=0.001, \varepsilon(t)=e^{-800 t}$

In Fig. 1 the problem of set point tracking of the closed-loop system is shown, while Fig. 2 indicates growth pattern (number of rules).

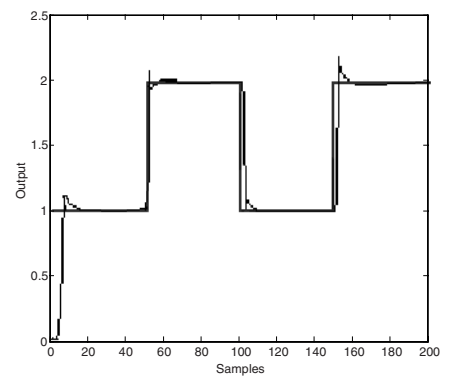

Fig. 1. Set point trajectory and output

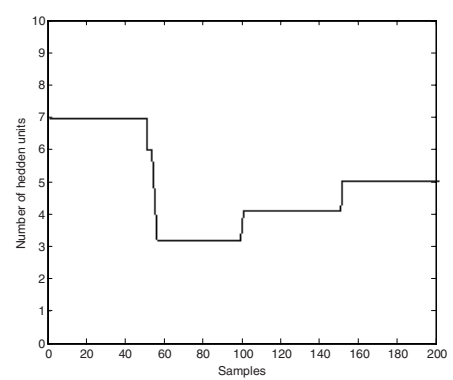

Fig. 2. Growth pattern (number of rules)

\section{Conclusion}

In this paper, firstly, we proposed a novel approach to train the TSWNN. An AFGLVQ technique was adopted to adjust the kernels of the TSWNN, and VRLS was applied to update the connection weights of the network. Secondly, on the basis of the one-step ahead TSWNN predictor, a numerically stable Davidon's least squares-based minimization approach was used to optimize the control law iteratively in each sample period. The simulations demonstrated the rapid learning and adaptive property of the identification algorithm and the effectiveness of control algorithm. 


\section{References}

1. Cao S.G., Rees N.W., Feng G. Analysis and design for a complex control systems, part I: fuzzy modeling and identification. Automatica, 6(8), pp.1017-1028, 1997.

2. Yingwei $\mathrm{Lu}$ and P. Saratchandran, Identification of time-varying nonlinear systems using minimal radial basis function neural networks, IEE Proc.: Cont. Theory and App.144(2), pp. 202-208, 1997.

3. Fabri S., and Kadirkamanatham V., Dynamic Structure Neural Networks for Stable Adaptive Control of Nonlinear Systems. IEEE Transaction on Neural Networks, 7(5), pp.1151-1167, 1996.

4. Jeffrey T.S. and Kevin M. Passino, Stable Adaptive Control Using Fuzzy Systems and Neural Networks, IEEE Transactions on Fuzzy Systems 4(3), pp.339-359, 1996.

5. Sanner R.M. and Jean-Jacques E. Slotine, Gaussian Networks for Direct Adaptive Control, IEEE Transaction on Neural Networks, 3(6), pp.837-867, 1992. 